\title{
Synchronization Phenomena in van der Pol Oscillators Coupled by Fifth-Power Nonlinear Resistor
}

\author{
Yoko Uwate $^{\dagger}$ and Yoshifumi Nishio ${ }^{\dagger}$ \\ $\dagger$ Dept. of Electrical and Electronic Engineering, \\ Tokushima University, 2-1 Minami-Josanjima, Tokushima, Japan \\ Email: \{uwate, nishio\}@ee.tokushima-u.ac.jp
}

\begin{abstract}
In this study, synchronization phenomena observed in van der Pol oscillators coupled by a fifth-power nonlinear resistor are investigated. By carrying out computer simulations, interesting synchronization phenomena can be confirmed to be generated in this system. Namely, the synchronization states change according to the coupling strength and nonlinearity of the coupling resistor.
\end{abstract}

\section{Introduction}

Synchronization phenomena in complex systems are very interesting to describe various higher-dimensional nonlinear phenomena in the field of natural science. Studies on synchronization phenomena of coupled oscillators are extensively carried out in various fields, physics [1]-[4], biology [5], [6] engineering [7]-[12] and so on. Because many researchers suggest that synchronization phenomena of coupled oscillators have some relations to information processing in the brain. We consider that it is very important to investigate the synchronization phenomena of coupled oscillators to realize a brain computer for the future engineering application.

On the other hand, there are some systems whose dissipation factors vary with time, for example, under the time-variation of the ambient temperature, an equation describing an object moving in a space with some friction and an equation governing a circuit with a resistor whose temperature coefficient is sensitive such as thermistor. However, there are few discussions about coupled oscillators coupling by a time-varying resistor.

In our previous research, we have investigated synchronization phenomena in van der Pol oscillators coupled by a time-varying resistor. We realized the timevarying resistor by switching a positive and a negative resistors periodically. By changing the duty ratio $p$, we confirmed that the characteristics of the synchronization phenomena changed as follows. First, for smaller $p$, the two coupled oscillators are synchronized only in anti-phase. Second, for intermediate $p$, the coexistence of the in-phase and the anti-phase synchroniza- tions can be observed. Finally, for larger $p$, only the in-phase synchronization can be confirmed.

Coexistence of attractors in coupled oscillatory systems is important for modeling of information processing mechanisms of the brain and applying them to novel parallel information processing systems. Generally, in two oscillators coupled by a resistor, only one synchronization state becomes stable even if plural states exist. In this study, two van der Pol oscillators coupled by a fifth-power nonlinear resistor are investigated. By carrying out computer calculations, interesting synchronization phenomena can be confirmed to be generated in this system. First, the coexistence of in-phase and anti-phase synchronization are observed. Next, we investigate the influence of the coupling strength and nonlinearity of the van der Pol oscillator.

\section{Circuit Model}

Figure 1 shows the circuit model. In this circuit, two identical van der Pol oscillators are coupled by a fifthpower nonlinear resistor. Synchronization phenomena for the case that the coupling resistor is a simple resistor have been investigated in [7],[8]. Namely, the in-phase synchronization is stable for a negative coupling resistor, while the anti-phase synchronization is stable for a positive coupling resistor.

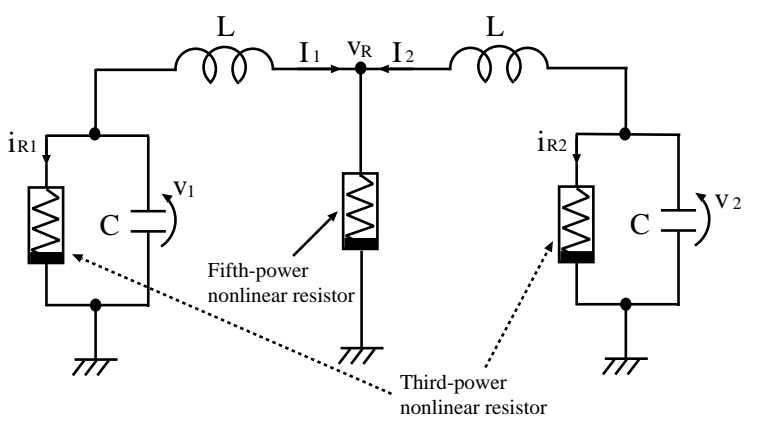

Figure 1: Circuit model.

In this study, we consider the fifth-power nonlinear resistor as coupling resistor. The characteristics of the 
fifth-power nonlinear resistor is defined as follows,

$$
v_{R}=g_{1}\left(I_{1}+I_{2}\right)-g_{3}\left(I_{1}+I_{2}\right)^{3}+g_{5}\left(I_{1}+I_{2}\right)^{5} .
$$

Next, the $v_{k}-i_{R k}$ characteristics of the third-power nonlinear resistor are defined as following equation.

$$
i_{R k}=-g_{1} v_{k}+g_{3} v_{k}^{3}
$$

By changing the variables and parameters,

$$
\begin{gathered}
v_{k}=\sqrt{\frac{g_{1}}{g_{5}}} \sqrt{\frac{L}{C}}, \quad i_{k}=\sqrt{\frac{g_{1}}{g_{5}}}, \quad t=\sqrt{L C} \tau, \\
\alpha=g_{1} \sqrt{\frac{L}{C}}, \quad \beta=\frac{g_{3}}{g_{1}} \sqrt{\frac{g_{1}}{g_{5}}} \\
\varepsilon=g_{1} \sqrt{\frac{C}{L}}, \quad \zeta=\frac{L}{C}
\end{gathered}
$$

the normalized circuit equations are given as

$$
\left\{\begin{aligned}
& \frac{d x_{k}}{d \tau}=-y_{k}+\varepsilon\left(x_{k}-\beta \zeta x_{k}^{3}\right) \\
& \frac{d z_{k}}{d \tau}=x_{k}-\alpha\left\{\left(y_{1}+y_{2}\right)-\beta\left(y_{1}+y_{2}\right)^{3}+\left(y_{1}+y_{2}\right)^{5}\right\} \\
&(k=1,2)
\end{aligned}\right.
$$

where $\alpha$ is the coupling factor and $\varepsilon$ is the strength nonlinearity.

\section{Synchronization Phenomena}

\subsection{In-Phase and Anti-Phase Synchronization}

We observed that the two coupled oscillators are synchronized in in-phase and anti-phase as shown in Figs. 2 and 3. These two synchronization states can be obtained by giving different initial conditions. The parameters of the van der Pol oscillator are fixed as $\varepsilon=0.4, \alpha=0.002, \beta=4.0, \zeta=4.0$.
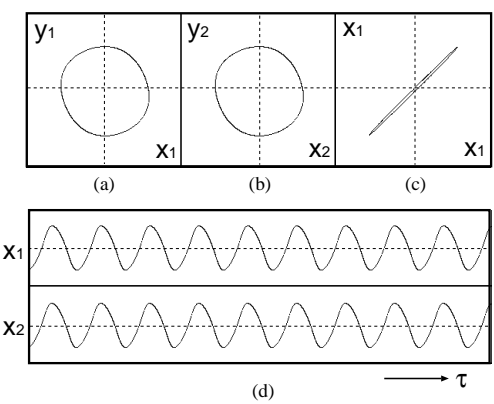

Figure 2: In-phase synchronization. (a) 1st circuit attractor $\left(x_{1}\right.$ vs $\left.y_{1}\right)$. (b) 2st circuit attractor $\left(x_{2}\right.$ vs $\left.y_{2}\right)$. (c) Phase difference $\left(x_{1}\right.$ vs $\left.x_{2}\right)$. (d) Time wave form $\left(\tau\right.$ vs $x_{1}$ and $\left.x_{2}\right) . \varepsilon=0.4$, $\alpha=0.002, \beta=4.0$ and $\zeta=4.0$.

Figure 4 shows the one parameter diagram of the phase difference. We can confirm the coexistence of in-phase and anti-phase synchronization around for $5.0<\beta<10.0$. Furthermore, we investigate the
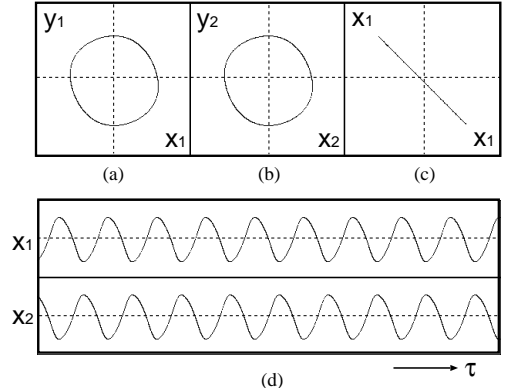

Figure 3: Anti-phase synchronization. (a) 1st circuit attractor $\left(x_{1}\right.$ vs $\left.y_{1}\right)$. (b) 2 st circuit attractor $\left(x_{2}\right.$ vs $\left.y_{2}\right)$. (c) Phase difference $\left(x_{1}\right.$ vs $\left.x_{2}\right)$. (d) Time wave form $\left(\tau\right.$ vs $x_{1}$ and $\left.x_{2}\right) . \varepsilon=0.4$, $\alpha=0.002, \beta=4.0$ and $\zeta=4.0$.

phase difference when the two parameters $\beta$ and $\zeta$ are changed. This simulated results are shown in Fig. 5. In this figure, the black color means the in-phase state and the yellow color means the anti-phase state. We can also observe the anti-phase state in these parameter area. Namely, the in-phase state area means the coexistence area. The coexistence area can be confirmed when $\beta$ are large values and $\zeta$ are small values.

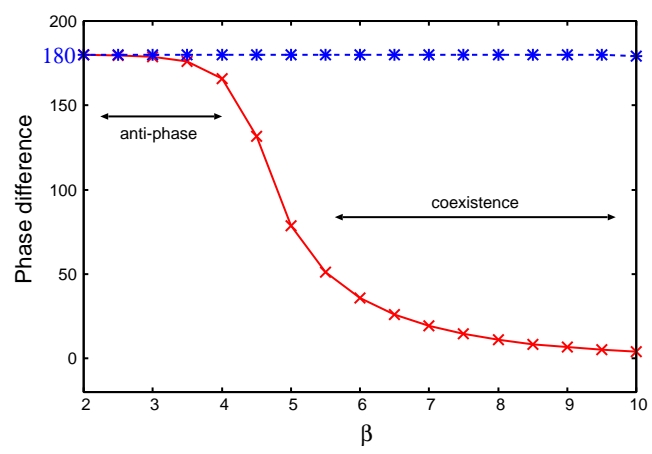

Figure 4: Phase difference for $\varepsilon=0.4, \alpha=0.002$ and $\zeta=5.0$.

\subsection{Synchronization in dependence on $\varepsilon$}

We investigate how the coexistence area depends on nonlinearity $\varepsilon$ of the van der Pol oscillator. The antiphase state is confirmed everywhere in these parameter area. So, the in-phase state area is corresponding to the coexistence area.

The computer simulation when $\varepsilon$ is fixed to 1.2 and 2.0 are shown in Fig. 6 (a), (b). The horizontal axis is $\beta$, the vertical axis is $\zeta$ and the black and yellow color denotes the the in-phase and the anti-phase states between the two coupled oscillators. In the case of weak nonlinearity $\varepsilon=1.2$, the in-phase state area can be occurred in $\zeta<5.0$ (Fig. 6 (a)). On the other hand, the case of $\varepsilon=2.0$, the in-phase state area is observed in $\zeta<7.0$ (Fig. 6 (b)). Figure 7 shows the area ratio of in-phase state when the nonlinear parameter $\varepsilon$ is changed. From this figure, we confirm that the in- 


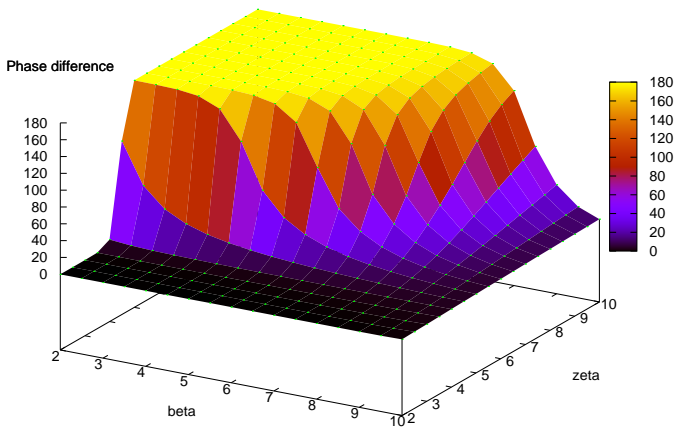

(a) 3D plot.

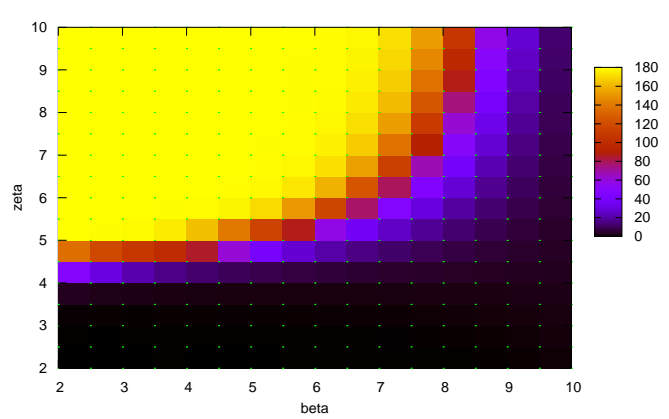

(b) $2 \mathrm{D}$ plot.

Figure 5: Phase difference for $\varepsilon=0.4$ and $\alpha=0.002$.

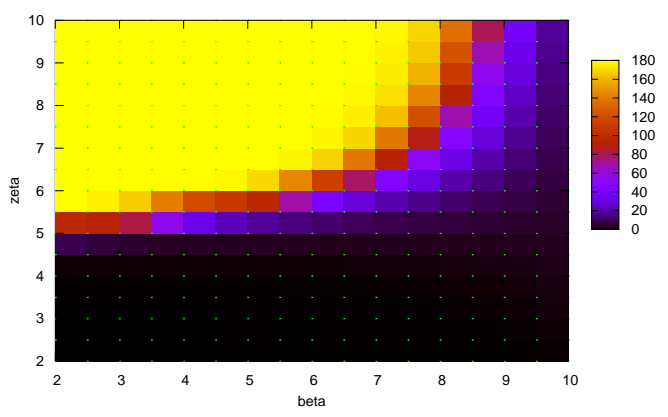

(a) $\varepsilon=1.2$.

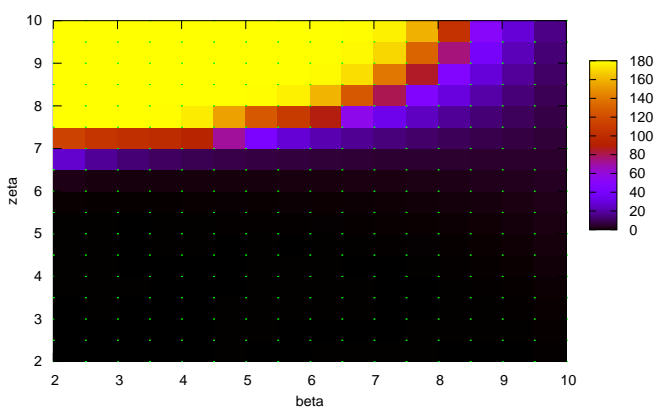

(b) $\varepsilon=2.0$.

Figure 6: Phase difference for $\alpha=0.002$.

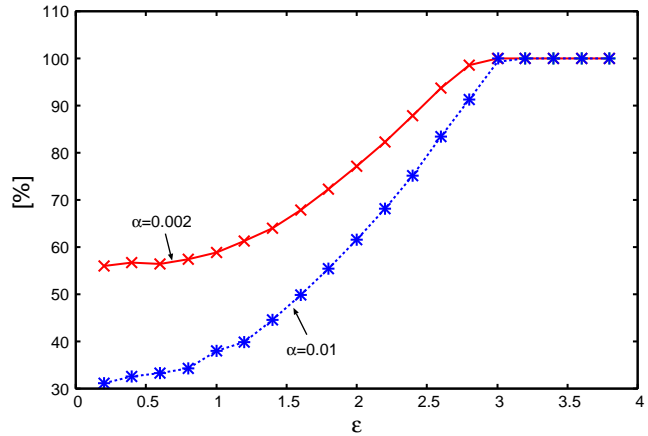

Figure 7: Area ratio of in-phase by changing $\varepsilon$.

phase (coexistence) area becomes large when the nonlinearity $\varepsilon$ of the van der Pol oscillator becomes large.

\subsection{Synchronization in dependence on $\alpha$}

Next, we investigate the coexistence area depends on the coupling strength $\alpha$. The computer simulation when $\alpha$ is fixed to 0.001 and 0.01 are shown in Fig. 8 (a), (b). In the case of small coupling strength, the in-phase state area can be occurred in $\zeta<6.0$ and $\beta>6.0$ (Fig. $8(\mathrm{a})$ ). On the other hand, the case of $\varepsilon=2.0$, the in-phase state area is observed in $\zeta<3.0$ (Fig. 8 (b)). Figure 9 shows the area ratio of in-phase state when the parameter $\alpha$ is changed. From this figure, we confirm that the in-phase (coexistence) area becomes small when the coupling strength $\alpha$ becomes large.

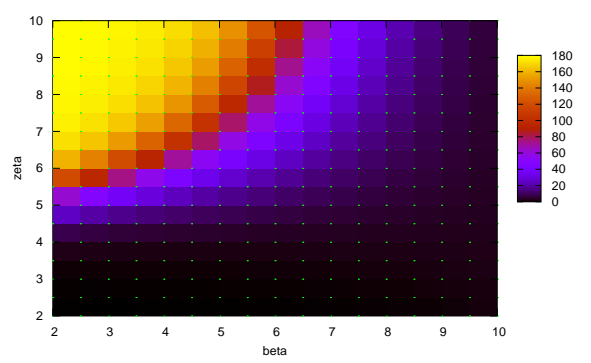

(a) $\alpha=0.001$.

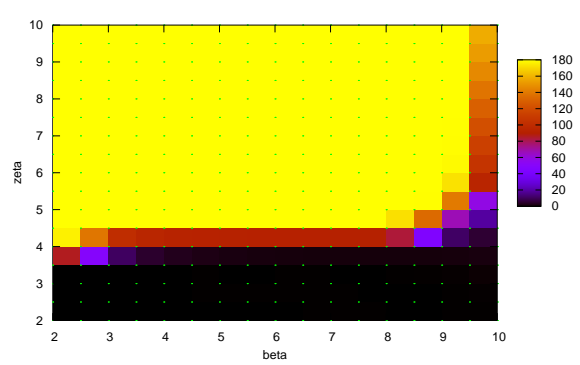

(b) $\alpha=0.01$.

Figure 8: Phase difference for $\varepsilon=0.4$. 


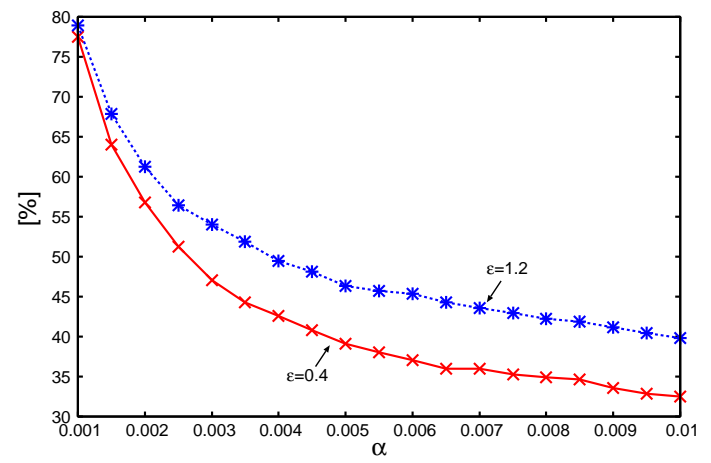

Figure 9: Area ratio of in-phase by changing $\alpha$.

\subsection{Amplitude of van der Pol Oscillator}

Finally, we investigate the amplitude of van der Pol oscillator when the two parameters $\beta$ and $\zeta$ are changed. The simulated result is shown in Fig. 10. We confirm that the amplitude of van der Pol oscillator becomes small when the both parameters $\beta$ and $\zeta$ increase.

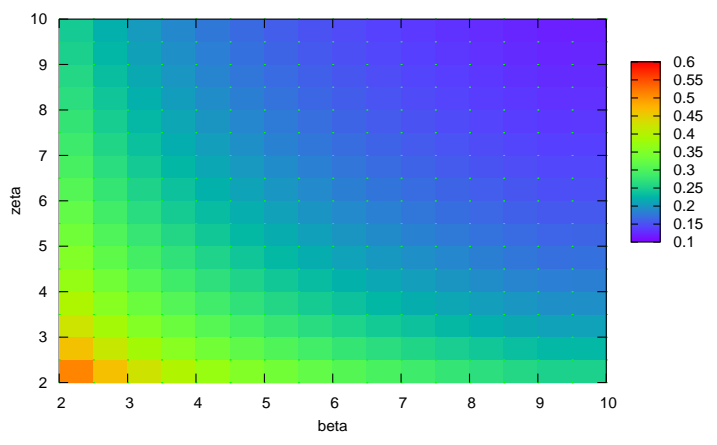

Figure 10: Amplitude of van der Pol oscillator for $\varepsilon=0.4$ and $\alpha=0.002$.

\section{Conclusions}

In this study, two van der Pol oscillators coupled by a fifth-power nonlinear resistor were investigated. By carrying out computer calculations, interesting synchronization phenomena could be confirmed to be generated in this system. First, the coexistence of inphase and anti-phase synchronization were observed. Furthermore, we have investigated the influence of the coupling strength and nonlinearity of the van der Pol oscillator.

\section{Acknowledgments}

This work was partly supported by Yazaki Memorial Foundation for Science and Technology.

\section{References}

[1] L.L. Bonilla, C.J. Perez Vicente and R. Spigler, "Timeperiodic phases in populations of nonlinearly coupled oscillators with bimodal frequency distributions," Physica D: Nonlinear Phenomena, vol.113, no.1, pp.7997, Feb. 1998.

[2] J.A. Sherratt, "Invading wave fronts and their oscillatory wakes are linked by a modulated traveling phase resetting wave," Physica D: Nonlinear Phenomena, vol.117, no.1-4, pp.145-166, June 1998.

[3] G. Abramson, V.M. Kenkre and A.R. Bishop, "Analytic solutions for nonlinear waves in coupled reacting systems," Physica A: Statistical Mechanics and its Applications, vol.305, no.3-4, pp.427-436, Mar. 2002.

[4] I. Belykh, M. Hasler, M. Lauret and H. Nijmeijer, "Synchronization and graph topology," Int. J. Bifurcation and Chaos, vol.15, no.11, pp.3423-3433, Nov. 2005.

[5] C.M. Gray, "Synchronous oscillations in neural systems: mechanisms and functions," J. Computational Neuroscience, vol.1, pp.11-38, 1994.

[6] J. Cosp, J. Madrenas, E. Alarcon, E. Vidal and G. Villar, "Synchronization of nonlinear electronic oscillators for neural computation," IEEE Trans. Neural Networks, vol.15, no.5, pp.1315-1327, Sep. 2004.

[7] T. Suezaki and S. Mori, "Mutual synchronization of two oscillators," Trans. IECE, vol.48, no.9, pp.15511557, Sep. 1965.

[8] H. Kimura and K. Mano, "Some properties of mutually synchronized oscillators coupled by resistance," Trans. IECE, vol.48, no.10, pp.1647-1656, Oct. 1965.

[9] S.P. Datardina and D.A. Linkens, "Multimode oscillations in mutually coupled van der Pol type oscillators with fifth-power nonlinear characteristics," IEEE Trans. Circuits Syst., vol.CAS-25, no.5, pp.308-315, May 1978.

[10] Y. Nishio and S. Mori, "Mutually coupled oscillators with an extremely large number of steady states," Proc. of ISCAS'92, vol.2, pp.819-822, May 1992.

[11] M. Yamauchi, M. Wada, Y. Nishio and A. Ushida, "Wave propagation phenomena of phase states in oscillators coupled by inductors as a ladder," IEICE Trans. Fundamentals, vol.E82-A, no.11, pp.2592-2598, Nov. 1999.

[12] B. Liu, X. Liu, G. Chen and H. Wang, "Robust impulsive synchronization of uncertain dynamical networks," IEEE Trans. Circuits Syst. I, vol.52, no.7, pp.14311441, July 2005.

[13] Y. Uwate and Y. Nishio, "Synchronization phenomena in van der Pol oscillators coupled by a time-varying resistor," International Journal of Bifurcation and Chaos (to appear).

[14] Y. Nishio and S. Mori, "Chaotic phenomena in nonlinear circuits with time-varying resistors," IEICE Trans. Fundamentals, vol.E76-A, no.3, pp.467-475, Mar. 1993. 